\title{
The Significance of Natural Anastomoses among Intracranial Vessels in Moyamoya Disease
}

W e read with great interest the article by Bonasia et $\mathrm{al}^{1}$ regarding the angiographic analysis of natural anastomoses between the posterior cerebral arteries (PCAs) and anterior cerebral arteries (ACAs) in Moyamoya disease (MMD) and syndrome. The authors observed 3 different types of anastomoses between the anterior and posterior circulations, with different abilities to compensate the anterior circulation. We really appreciate the interesting observations in their Conclusions. Meanwhile, after reading this article, we would like to highlight 2 important questions that it raises.

First, the authors found a wide anastomoses among intracranial vessels, especially between the PCAs and ACAs, in patients with MMD. This finding is consistent with our latest observations. ${ }^{2}$ We observed different hemodynamic sources of the recipient parasylvian cortical arteries (PSCAs) among the frontal, temporal, and parietal PSCAs in MMD hemispheres. PSCAs from the PCAs accounted for $25.3 \%$. The extensive anastomosis between PCAs and ACAs provides new evidence to explain the various hemodynamic sources of PSCAs observed in MMD hemispheres. More interesting, in the current study, the degree of posterior collaterals among Suzuki scores was positively correlated. We observed the same phenomenon. This suggests that although the posterior circulation is less affected in MMD, the compensatory effect of the posterior circulation on the anterior ischemic and hypoperfusion areas becomes more and more important as the disease progresses.

Second, the broad anastomosis of intracranial blood vessels in patients with MMD reminds neurosurgeons that the hemodynamics in PSCAs are complex. Previous studies ${ }^{3,4}$ have also

http://dx.doi.org/10.3174/ajnr.A6502 mentioned the different types of collaterals that naturally develop in MMD. These collaterals can not only provide blood flow support for the ischemic area but also are risk factors for hemorrhagic stroke. During direct bypass surgery, surgeons should pay more attention to DSA, rather than relying solely on anatomy, to determine the hemodynamic source of the recipient vessel. Recipient vessels of different hemodynamic sources have different effects on postoperative perfusion recovery in different cerebral regions. We have found that the hemodynamic source of the recipient vessel is closely related to postoperative hyperperfusion syndrome.

\section{REFERENCES}

1. Bonasia S, Ciccio G, Smajda S, et al. Angiographic analysis of natural anastomoses between the posterior and anterior cerebral arteries in Moyamoya disease and syndrome. AJNR Am J Neuroradiol 2019;40:2066-72 CrossRef Medline

2. Zhang J, Li S, Fujimura M, et al. Hemodynamic analysis of the recipient parasylvian cortical arteries for predicting postoperative hyperperfusion during STA-MCA bypass in adult patients with Moyamoya disease. J Neurosurg 2019 Dec 27:1-8. [Epub ahead of print] CrossRef Medline

3. Baltsavias G, Khan N, Valavanis A. The collateral circulation in pediatric Moyamoya disease. Childs Nerv Syst 2015;31:389-98 CrossRef Medline

4. Robert T, Ciccio G, Sylvestre P, et al. Anatomic and angiographic analyses of ophthalmic artery collaterals in Moyamoya disease. AJNR Am J Neuroradiol 2018;39:1121-26 CrossRef Medline 